\title{
THE USE OF AVERTIN FOR THE PRODUCTION OF BASAL NARCOSIS IN CHILDREN
}

\author{
BY \\ H. K. ASHWORTH, M.B., M.R.C.S., D.A., \\ Anaesthetist to the Manchester Royal Infirmary.
}

The introduction of avertin has been described as the most important discovery in anaesthesia for many years ${ }^{1}$, and one of the greatest of the benefits which it confers is the complete amnesia provided by this substance for children about to be submitted to the otherwise terrifying ordeal of inhalation anaesthesia. All observers are agreed that the psychological trauma inflicted on the patient by the ordeal of an operation is naturally greater in the case of children than in the majority of adults. The attendant circumstances of the induction of inhalation anaesthesiai are obviously the cause, this being the only part of the proceedings of which the patient is conscious. This psychological trauma may be hidden, butt it is soon apparent when-sometimes many years later-a child becomes aware that it is again to be subjected to the induction of inhalation anaesthesia. An anaesthetist witnesses few scenes more disturbing to all concerned than the abject terror so often displayed by the unfortunate child in these circumstances. Before the introduction of basal narcosis, the child was frequently submitted to the ordeal of being suddenly taken from his accustomed ward and confronted with the strange surroundings and strange faces in the anteroom of the theatre, followed by varying degrees of apprehension until he received the crowning horror of feeling suffocated by the anaesthetic mask placed over his face. The introduction of basal narcosis by means of methods both reliable and safe has made it possible entirely to abolish this undesirable accompaniment of operation. It is, in fact, hardly an overstatement to say that, excepting in case of urgency, or other exceptional circumstances, no conscious child should ever see the theatre or be submitted to the terrors associated with the induction of inhalation anaesthesia.

Although the psychological aspect is, in the case of children, obviously pre-eminent, it is by no means the only benefit conferred by the use of basal narcosis. Other advantages are:-

(1) Subsequent full surgical anaesthesia can often be maintained by the use of gas and oxygen alone, or, if necessary, with a greatly reduced amount of ethyl chloride or ether.

(2) The post-operative period is one of much greater comfort and wellbeing for the patient. Amnesia often persists for several hours after operation, although the patient will often take drinks and talk apparently quite rationally during this period. 
(3) The reduced amount of ether used tends to diminish the occurrence of post-operative vomiting and of respiratory complications of anaesthesia.

\section{Choice of drug.}

Many drugs are commonly used to produce basal narcosis, chiefly avertin, paraldehyde, or one of the barbiturates (nembutal, evipan or pernocton). Of these, avertin stands pre-eminent, particularly: for children. The comparative advantages of avertin may be summarized as follows :-

(1) Avertin in ordinary accepted dosage (0.075-0.1 gm. per kgm. bodyweight) is particularly safe for children, because they tolerate the drug extremely well. Avertin constantly produces a greater depth of narcosis in adults than in children. This tolerance has recently been confirmed by Boyd $^{2}$ who claims to give avertin safely in massive doses (up to $0.2 \mathrm{gm}$. per kgm.) to children in order to produce full surgical anaesthesia, a procedure unanimously condemned by leading authorities in this country ${ }^{3,4}$.

(2) Unlike many of the barbiturates (unless the latter are administered by the intravenous route, which is usually difficult in children under the age of five years) avertin always produces complete amnesia. The action of avertin is certain, and largely uniform, whereas that of the barbiturates, e.g., nembutal by mouth or by rectum, is uncertain and capricious.

(3) Restlessness during the induction of unconsciousness with avertin is uncommon. Loss of consciousness is unattended by any unpleasant subjective sensations, and the patient presents the appearance of natural sleep, rarely moving from the position adopted for the administration of the avertin.

(4) Post-operative restlessness after avertin is less common, particularly in the case of children, than after the use of a barbiturate.

(5) Avertin has little appreciable effect on the chief organs of excretion, i.e., the liver and kidneys. Too much unwarranted prejudice still exists against avertin on this score, but with the increasing experience that has been gained since the introduction of the drug to England in 1929, the greater is the tendency of authoritative opinion, based upon both clinical ${ }^{5},{ }^{6}$, and experimental ${ }^{7,8,9}$ evidence, to regard the toxic effects on the excretory organs as negligible. It is, for instance, as yet insufficiently realized that avertin is less toxic than ether, and is completely non-irritating to the respiratory passages.

(6) Avertin, unlike paraldehyde, has no appreciable odour at a distance of more than a few inches from the flask containing the solution. Paraldehyde is still widely used as a basal narcotic for children, largely on account of the reputed safety of the drug. The writer's experience of paraldehyde is small, because it does not appear necessary to subject the attendants of the patient to the unpleasant, nauseating odour of this drug when avertin provides an equally safe or safer alternative. Moreover, the action of paraldehyde is less uniform than that of avertin, and many cases have been reported in which alarming signs of undue respiratory depression have developed in the post-operative period ${ }^{10,11}$.

(7) Avertin is rapidly absorbed from the lower bowel ${ }^{12}$, which relieves the attendants from the necessity of washing-out any remaining solution after the patient has returned to bed.

There is no doubt that the use of avertin has largely superseded that of paraldehyde for adults, and the next few years will probably show the same trend in the case of children. 
Avertin has two disadvantages: (1) The correct preparation of the solution requires time and care $^{13}$. (2) If the condition of the patient, following a severe operation, is such that rectal salines are indicated, these are not well retained during the first four or five post-operative hours. This disadvantage is common to all narcotics administered per rectum.

\section{Contra-indications.}

There are three definite contra-indications to the use of avertin:(1) Whenever it is impracticable either to obtain or accurately to estimate the weight of the patient; (2) the presence of pathological conditions of the rectum or colon; and (3) operations on the rectum or colon. Two relative contra-indications which are subjects of controversy among leading authorities are:-(1) The presence of 'moist' breath-sounds; and (2) impaired liver function. Sir Francis Shipway states that the presence of bronchitis is not a contra-indication for the use of avertin ${ }^{14}$. Although the writer has administered avertin without harm on three occasions to patients suffering from active bronchiectasis, his inclination is to deprecate the use of avertin under such conditions, as tending to delay the rapid return of the maximum respiratory efficiency so desirable in such cases. In spite of contrary expression of opinion ${ }^{15}$, the trend of clinical evidence seems increasingly to be that avertin may be used with safety even in the presence of grossly damaged liver function ${ }^{16,17}$.

Avertin plus nitrous oxide and oxygen have recently been successfully administered to a boy aged twelve years for the purpose of a resection of his splanchnic nerves in an effort to mitigate an uncontrollably severe diabetic state. On the morning of operation his blood-sugar content was $170 \mathrm{mgm}$. per 100 c.c. Operation was performed in the afternoon. In the evening his blood-sugar content was $150 \mathrm{mgm}$. per 100 c.c. During the following forty-eight hours, the blood-sugar content was as follows:-

\begin{tabular}{|c|c|c|c|c|c|c|c|}
\hline Ist morning & $\cdots$ & & & 135 & mgm. & per & 100 \\
\hline 1st evening & $\cdots$ & $\ldots$ & $\ldots$ & 145 & , & ," & , \\
\hline 2nd morning & ${ }^{\circ}$ & $\cdots$ & $\ldots$ & 140 & , & , & ," \\
\hline 2nd evening & $\ldots$ & $\cdots$ & $\cdots$ & 70 & ,, & ," & , \\
\hline
\end{tabular}
avertin.

This case provides a particularly striking example of the low toxicity of

The conclusion has been reached, based on personal experience of nearly 1,000 cases, that, except in the presence of the graver emergencies of surgery, if a patient will tolerate any anaesthetic at all, avertin may always be given with confidence and safety.

\section{Technique of administration.}

Although the following description applies particularly to the use of avertin for routine sessions for operations for the removal of tonsils and adenoids, it is equally applicable in individual cases for other operations. It is based on a series of $\mathbf{5 , 9 1 8}$ administrations at the Booth Hall Hospital, Manchester, where this method has been employed for the last six years. 
Preparation of the patient.-The children are admitted the day before the operation. In non-abdominal cases, unless the parent reports that the child suffers from obstinate constipation, no aperient is necessary. A simple enema is administered about $6 \mathrm{p.m}$. The evening meal should include glucose, either in the form of jam or syrup, or, alternatively, each child may be given $\frac{1}{4} \mathrm{lb}$. of boiled sweets. Four hours before operation a simple rectal wash-out is administered. Three hours before operation each child receives a plate of porridge or gruel, together with a liberal' helping of syrup.

Preparation and dosage of avertin solution.-The weight of each patient should be entered in writing on the temperature chart, and the name, weight and required dosage of avertin for each patient should be sent to the dispensary in writing. Telephonic or verbal transmission of these particulars should be prohibited in order to prevent mistakes. The usual dosage for cases in which operations are to be undertaken on the air passages, and in which a rapid return of the cough-reflex is consequently desirable, is 0.075 or $0.08 \mathrm{gm}$. per $\mathrm{kgm}$. body weight. For operations other than those on the respiratory passages, a full dose of $0.1 \mathrm{gm}$. per $\mathrm{kgm}$. should be prescribed. Avertin is administered as a 2.5 per cent. solution in distilled water. The description of the actual preparation of the avertin solution is outside the scope of this paper, and may be obtained elsewhere (Bayer Products, Ltd.), but the recommended method should be strictly adopted. For a long list of cases, in which each patient receives the same dosage of avertin (e.g. all $0.075 \mathrm{gm}$. per kgm. body weight) the simplest method of procedure is to prepare in bulk the total quantity of avertin solution required, and then to measure out from this the requisite amount for each child. The required solution can be prepared in fifteen minutes. The bottle containing the prepared solution for each patient should bear a label stating the name and weight of the patient, the quantity of avertin fluid and water used, and the date and hour of preparation. These particulars should be checked by the ward-nurse against those on the temperature chart before the actual administration is begun. These precautions may seem to be unnecessarily severe, but rigid adherence to these rules is the only adequate safeguard against the possibly disastrous results of administering the wrong dose to the wrong patient.

Administration.- Thirty minutes before the time of operation, the child is placed in the left lateral position, and the solution is run slowly into the rectum by means of a small catheter, tube and funnel. Administration should be slow, taking up to ten minutes to complete. Immediately afterwards, the child should receive a dose of atropine, graduated according to age as follows:-

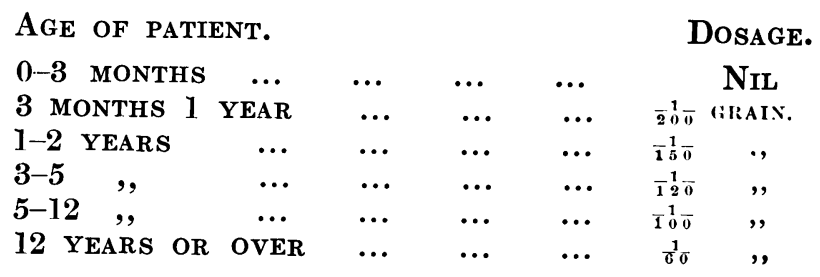

The child should then be left quiet, when it usually drops off to sleep within fifteen minutes, and often before the administration is completed. It is then transported to the ante-room of the operating theatre to await operation. At this stage, it can easily be roused by light stimulation, such as pinching the skin, and will invariably resist the application of a mask when the administration of ethyl chloride or ether is begun, but amnesia is always complete, 


\section{Surgical anaesthesia after premedication with avertin.}

The administration of ethyl chloride or somnoform-preferably by means of a bag-suffices for the production of surgical anaesthesia for the removal of tonsils and adenoids by the ' rapid' technique. Induction of anaesthesia by this method is rapid, and the face-piece, or mask, should be removed as soon as the child has taken three stertorous inspirations. One of the great advantages of avertin is to increase the time available for the performance of the operation after the face-piece or mask has been removed. For other surgical operations, or for more leisurely removal of tonsils, including, if necessary, the insertion of a Boyle-Davies gag, anaesthesia should be induced by means of an ethyl chloride-ether sequence administered on an open mask, and anaesthesia subsequently maintained with ether, or, for procedures other than intra-abdominal or oral operations, with nitrous oxide and oxygen.

The only disadvantage of the use of avertin is that the amount of haemorrhage at the operation is slightly increased. This, however, is a temporary, and therefore unimportant phenomenon, as is proved by the fact that the incidence of reactionary haemorrhage is not increased.

\section{Post-operative treatment.}

The safety of this method of anaesthesia depends on scrupulous attention to the following points after the operation is concluded:-

(1) The child must not be removed from the operating theatre until the cough reflex has returned. If the ' rapid' technique has been used, this reflex usually returns within one minute of the conclusion of the operation.

(2) The child must not be left with the airway unattended during transport back to the ward.

(3) After return to the ward, the same precautions regarding the airway must be observed until all danger of obstruction is past.

The use of a ' recovery' room adjacent to the operating theatre is of enormous value in facilitating the post-operative care of all patients who have undergone anaesthesia. It may be thought that this procedure involves undue loss of time, and necessitates the presence of a large number of attendants, but with careful organisation such is not the case. It is not necessary to have one nurse for each child. By giving avertin to the first child fifty minutes before operation (the exact time of administration is not important provided that it is within the limits of fifty to thirty minutes before operation), three or four children may be transported on one trolley, in charge of one nurse, to await operation in the ante-room. After the operation, the same procedure may be adopted, and the children re-transported to the ward in batches of two or three. The use of avertin abolishes the necessity for segregating the children awaiting operation in a separate ante-room, and thus eases the task of the supervising nurses. 


\section{Complications following the use of avertin.}

Although, with a single exception, no complications following the usc of avertin have occurred in this series of cases, no description would be complete without a reference to the signs and treatment of an overdosc. These are those common to all narcotic drugs which, in large doses, cause a progressive paralysis of the medullary centres-profound unconsciousness, slow and shallow respirations, cyanosis, slow and feeble pulse, fall in body temperature, contraction of the pupil, and absence of the eye reflexcs. Treatment consists of the application of warmth, administration (by mcans of an endo-tracheal catheter, if available) of 10 per cent. $\mathrm{CO}_{2}$ in oxygen, and the intravenous injection of $2-3$ c.c. of coramine, which is stated to be almost specific as an antidote ${ }^{18}$.

There was one death in the series of cases under review.

A. K. was admitted for removal of tonsils and adenoids. He was just thirteen years of age, but weighed 10 st. $10 \frac{1}{2} \mathrm{lb}$. and received $5 \cdot 3$ c.c. of avertin. The boy went to sleep in the usual way after being given avertin at 3 o'clock in the afternoon, operation taking place about 4 o'clock. At 5.45 haemorrhage occurred when he was seen by the operator and the medical superintendent. He was seen again at 7 p.m. when he was not showing the usual signs of returning consciousness. At $8 \mathrm{p.m}$. he was cyanosed and his respirations were reduced in frequency. No improvement taking place, at 9.30 oxygen and carbon dioxide were administered, and the anaesthetist was sent for, oxygen and carbon dioxide then being given by intra-tracheal catheter, and artificial respiration resorted to for half an hour with considerable improvement. The respiration rate returned to normal. He then for the first time showed signs of returning consciousness. Whilst in the theatre he had an intravenous gum saline administered with the usual cardiac and respiratory stimulants with a rectal wash-out. $\Delta$ liver preparation was also used. At 9.30 a.m. on the following day the pulse was good in rate and volume, the respiratory rate normal, but the patient had not recovered consciousness. Further cardiac stumulants were injected. At 11 a.m. respiration failed, and death took place at noon, twenty-one hours after the administration of avertin. At the post-mortem examination the pituitary gland showed some signs of enlargement and the pathologists's report was:- 'The gland appeared to be slightly larger in size than normal. Towards one side there was a paler area, possibly of slightly firmer consistence than the rest of the gland. On microscopical examination the anterior pituitary substance was normal in appearance with no localized overgrowth of any type of cell. There is probably some degree of diffuse hyperplasia but no definite adenoma and no tuberculosis.'

The writer is indebted to Dr. J. D'Ewart, Medical Superintendent of the Booth Hall Hospital, Manchester, both for so kindly placing at his disposal all the valuable material and knowledge obtained from a study of this series of cases, and also for ever-ready help and advice in the actual preparation of this paper. 


\section{REFERENCES.}

1. Shipway, Sir F. E., Personal communication.

2. Boyd, J., Brit. Med. J., London, 1935, i, 1120.

3. Blomfield, J., \& Shipway, Sir F. E., Proc. Roy. Soc. Med., London, 1929,

4. Hewer, C. Langton, Recent Advances in Anaesthesia, London, 1932, 34.

5. Ashworth, H. K., Brit. Med. J., London, 1932, i, 1123.

6. Shipway, Sir F. E., Brit. J. Anaesth., Manchester, 1935, XII, 157.

7. Widenhorn, H., Anaesth. \& Analg., Elmira, 1932, XI, 60.

8. Pitt, N. E., Lancet, London, 1935, i, 741.

9. Van Zyl, F. D. du T., South African Med. J., Cape Town, 1933, VII, 579.

10. Williams, R. E., Personal communication.

11. Brown, G., Brit. J. Anaesth., Manchester, 1935, XIII, 25.

12. Blomfield, J., \& Shipway, Sir F. E., Lancet, London, 1929, i, 546.

13. Idem, ibid.

14. Shipway, Sir F. E., Brit. J. Anaesth., Manchester, 1935, XII, 156.

15. Hewer, C. Langton, Recent Advances in Anaesthesia, London, 1932, 34.

16. Shipway, Sir F. E., Brit. J. Anaesth., Manchester, 1935, XII, 162.

17. Ashworth, H. K., Brit. Med. J., London, 1932, i, 1123.

18. Kennedy, W. P., Lancet, London, 1932, i, 1143. 